\title{
One-Pot Synthesis of Oxidation-Sensitive Supramolecular Gels and Vesicles
}

\author{
Aroa Duro-Castano,* Laura Rodríguez-Arco, Lorena Ruiz-Pérez, Cesare De Pace, Gabriele Marchello, \\ Carlos Noble-Jesus, and Giuseppe Battaglia*
}

Cite This: Biomacromolecules 2021, 22, 5052-5064

Read Online

ACCESS | Lill Metrics \& More | 回 Article Recommendations | sl Supporting Information

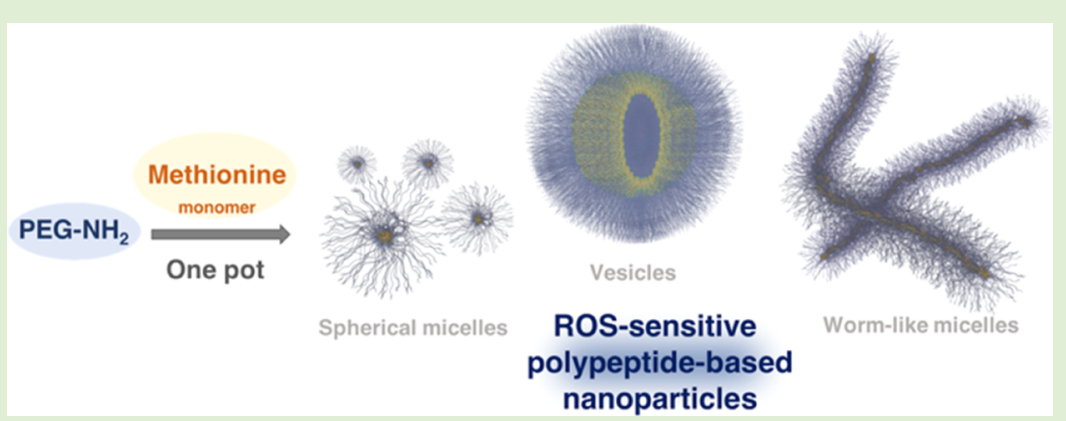

ABSTRACT: Polypeptide-based nanoparticles offer unique advantages from a nanomedicine perspective such as biocompatibility, biodegradability, and stimuli-responsive properties to (patho)physiological conditions. Conventionally, self-assembled polypeptide nanostructures are prepared by first synthesizing their constituent amphiphilic polypeptides followed by postpolymerization selfassembly. Herein, we describe the one-pot synthesis of oxidation-sensitive supramolecular micelles and vesicles. This was achieved by polymerization-induced self-assembly (PISA) of the N-carboxyanhydride (NCA) precursor of methionine using poly(ethylene oxide) as a stabilizing and hydrophilic block in dimethyl sulfoxide (DMSO). By adjusting the hydrophobic block length and concentration, we obtained a range of morphologies from spherical to wormlike micelles, to vesicles. Remarkably, the secondary structure of polypeptides greatly influenced the final morphology of the assemblies. Surprisingly, wormlike micellar morphologies were obtained for a wide range of methionine block lengths and solid contents, with spherical micelles restricted to very short hydrophobic lengths. Wormlike micelles further assembled into oxidation-sensitive, self-standing gels in the reaction pot. Both vesicles and wormlike micelles obtained using this method demonstrated to degrade under controlled oxidant conditions, which would expand their biomedical applications such as in sustained drug release or as cellular scaffolds in tissue engineering.

\section{INTRODUCTION}

Polypeptides are the backbone of biological organization and, as such, have attracted considerable interest from organic chemists to synthesize them. The most established approach is based on solid-phase methods that allow the synthesis of complex sequences, which are never larger than tens of amino acids. On the other end, long polypeptides without sequence control can be made by ring-opening polymerization of $\mathrm{N}$ carboxyanhydrides (NCAs). ${ }^{1}$ Additionally, this technique allows for easy modulation of the macromolecular engineering. ${ }^{2}$ The most important features of polymeric biomaterials, i.e., biocompatibility and biodegradability, often provided by stimuli-responsiveness, ${ }^{1}$ can be achieved with single amino acid peptides. Stimuli-responsiveness allows for nanoparticle disruption/degradation upon specific physiological conditions such as $\mathrm{pH}$ or redox. ${ }^{3}$ Indeed, $\mathrm{pH}$ sensitivity can be attained using natural amino acids containing (de)protonable residues such as glutamic and aspartic acids, lysine, or histidine, while redox-responsiveness is given by amino acids like cysteine or methionine. ${ }^{3}$ In addition, custom-built stimuli-responsiveness is straightforwardly available by conjugating stimulus-specific moieties to the polypeptide backbone (i.e., sugars, peptide sequences, etc.), thus giving access to further triggers. ${ }^{3}$ Additionally, from a manufacturing perspective, polypeptides hold attractive self-assembly behaviors owing to a higher control over intra- and interchain interactions, altogether with adjustable secondary and tertiary structures, as their protein analogues. ${ }^{4}$ Indeed, amphiphilic diblock copolypeptides comprising hydrophilic and hydrophobic sequences have been shown to self-assemble in a variety of structures. ${ }^{5}$ All of these properties make polypeptides ideal candidates for

Received: August 10, 2021

Revised: September 17, 2021

Published: November 11, 2021 
(a)

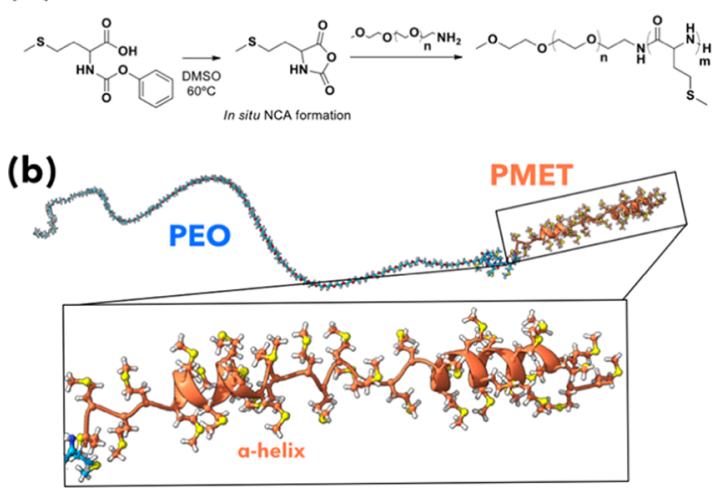

(c)

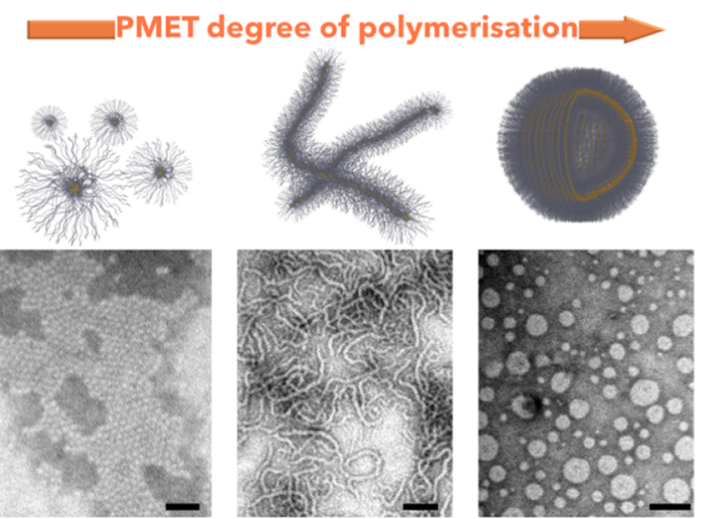

Figure 1. Schematic representation of the "one-pot" synthesis. Synthesis scheme for the ring-opening polymerization (ROP) of activated urethane of methionine in DMSO as solvent (a). Molecular model of the PEO-PMET diblock copolymer showing the partial folding of the polypeptide (b). Scheme showing the evolution from spherical micelles to cylindrical micelles to vesicles as a function of the PMET degree of polymerization with relative transmission electron micrographs $(\mathrm{c})$. Scale bar $=100 \mathrm{~nm}$.

translation in many biological applications ranging from drug/ gene delivery, imaging, or tissue engineering. ${ }^{6-9}$

Traditionally, self-assembled structures using polypeptides are prepared by first synthesizing their constituent amphiphilic polypeptides (i.e., unimers), followed by postpolymerization self-assembly using a variety of techniques such as film rehydration, solvent switch, $\mathrm{pH}$ adjustment, nanoprecipitation, salting-out, electroformation, layer-by-layer assembly, and microfluidic techniques. ${ }^{10,11}$ However, most of these selfassembly methods only allow for diluted nanoparticle dispersions $(<1 \%$ solid content, solid content being an expression of the polymer concentration at the end of the reaction). Understandably, limitations in scaling-up processes and manufacture hamper the clinical translation and commercial applications of polypeptide-based assemblies. Consequently, there is a clear need for a suitable and scalable method of polypeptide nanoparticle production. In this regard, polymerization-induced block copolymer self-assembly (PISA) arises as a key solution. ${ }^{12,13}$ PISA comprises the in situ growth of a "living" amphiphilic polymer chain and its simultaneous self-assembly. ${ }^{14}$ Quite uniquely, PISA allows us to operate at high solid contents to generate a plethora of homogeneous and reproducible structures such as spheres, worms, and vesicles. ${ }^{15,16}$ For PISA to work, a solvent-soluble macroinitiator is generally used to polymerize a solvent-soluble monomer, resulting in a solvent-insoluble polymer block, which drives the self-assembly process. ${ }^{17}$ To date, the PISA approach has been widely explored in the synthesis of polymethacrylate derivatives using controlled radical polymerization processes in dispersion or emulsion. ${ }^{18}$ However, there are only a few examples in the literature exploiting ring-opening polymerization (ROP)-induced self-assembly. ${ }^{19-24}$ Toward this aim, Jiang et al. recently reported the formation of poly(ethylene oxide)polyphenylalanine vesicles in tetrahydrofuran by what was referred to as NCA-PISA. ${ }^{25}$ In a similar manner, Lecommandoux and Bonduelle reported the formation of needle-like particles from poly(ethylene oxide)poly(benzylglutamate) by aqueous NCA-PISA, renamed this time as ROPISA. ${ }^{26}$ Although undoubtedly novel, both methods report only a maximum of 40 units of the hydrophobic chain domain and are limited to the use of diluted polymer concentrations of maximum $13 \%$ solid content, probably due to the well-known sensitivity of the NCA monomers to hydrolysis. $^{27,28}$ In addition, one single nanoparticle morphol- ogy was reported in each of these two investigations, i.e., vesicles or needle-like structures. These restrictions might reduce access to the production of other nanoparticle morphologies (which can dictate, for example, the rate of endocytosis) and could represent a potential hurdle for the scale-up production of peptide-based nanoparticles. Therefore, new synthetic strategies which allow the translation of polypeptide-based formulations of well-defined morphologies from laboratory to market, are strongly desired. Finally, another key requirement for the translation of synthetic nanomedicines and biomaterials into clinical use is their ability to trigger their functional output (e.g., cargo delivery) under the specific physiological conditions of the diseased/ malfunctioning site. We propose here the use of reactive oxygen species (ROS) connected to mitochondria dysfunction. ${ }^{29}$ Indeed, chronic oxidative stress is linked to many pathologies including atherosclerosis, neurodegeneration, arthritis, diabetes, inflammation, cancer, and metastasis. ${ }^{30-34}$ Therefore, the unique redox microenvironment under these pathological conditions makes the affected regions different from their surroundings. For this reason, the use of building blocks responsive to ROS has been extensively investigated and exploited in nanomedicine to specifically deliver drugs, bioactive/imaging agents to the disease site as well as in prodrugs. ${ }^{35-37}$ In addition, ROS-mediated biodegradability under physiological conditions is also desirable to enhance biocompatibility of the biomaterials/nanomedicines.

In this work, we present a novel method for the one-pot production of multiple nanoparticle morphologies by dispersion polymerization of NCA precursors in aprotic solvents. The proposed methodology eliminates the risk of instability of NCAs with moisture/water that would otherwise lead to rapid hydrolysis, and therefore, the methodology presents no limits in solid content nor hydrophobic block length to obtain welldefined particles. Indeed, by adjusting the degree of polymerization of the hydrophobic block and the solid content, it is possible to engineer a wide range of structures (Figure 1). The employed building hydrophobic blocks are based on the ROSresponsive amino acid methionine, as an exemplary hydrophobic block, which makes the nanoparticles oxidationsensitive. $^{38}$ Remarkably, we also in situ monitored the degradation of these nanoparticles at the nanoscale via stateof-the-art liquid-phase electron microscopy. Nonetheless, our methodology could be extended to other amino acids, as 
shown in experiments involving phenylalanine as the hydrophobic block, thus proving its versatility. Finally, our synthetic approach can also be engineered to generate supramolecular hydrogels in the reaction pot, with potential future in biomedical applications such as wound healing, tissue regeneration, cell matrix mimics, and therapeutics in tissue engineering, biosensors, as well as in sustained drug release or antibacterial applications. ${ }^{39}$

\section{EXPERIMENTAL SECTION}

Materials. All chemicals were of reagent grade, obtained from Sigma-Aldrich (Dorset, U.K.), and used without further purification unless otherwise indicated. All solvents were obtained from SigmaAldrich (Dorset, U.K.). Those used in purifications were of analytical grade and used without further modifications except for anhydrous dimethyl sulfoxide used in the synthesis. Deuterated trifluoroacetic acid (TFA- $d$ ) was purchased from Fluorochem (U.K.). Methoxypoly(ethylene glycol)amine MW $5 \mathrm{kDa}$ was obtained from Iris Biotech GmbH (Marktredwitz, Germany). Dialysis was performed using Spectra/Por 6 Dialysis Tubing, $3.5 \mathrm{kDa}$ MWCO, $45 \mathrm{~mm}$ Flatwidth from Fisher Scientific (U.K.).

Polymerization-Induced Self-Assembly in Aprotic Solvent. The hydrophobic monomer precursor exemplary used in this study was an active urethane derivative precursor of the methionine amino acid, which cyclizes in situ to form an NCA reagent with increasing temperature. Such a precursor was synthesized in a previous step according to Yamada et al. ${ }^{40}$ Next, the monomer and the hydrophilic macroinitiator methoxy-poly(ethylene glycol)amine were weighed in a Schlenk flask provided with a stirrer bar and a sub-seal stopper. The mixture was subjected to three high-vacuum/argon cycles to remove any residual moisture. The feed ratio of initiator/monomer depended on the desired hydrophobic/hydrophilic ratio in the final synthesized polymer, being typically, 1 the initiator, and $x$ the equivalents of monomer according to the desired hydrophobic length. The reaction was performed using Schlenk techniques under argon atmosphere. The corresponding amount of the aprotic polar solvent (DMSO) according to the desired percentage of solid content (\% w) was then added. The reaction was left to proceed at $60{ }^{\circ} \mathrm{C}$ under vigorous stirring and argon atmosphere with an outlet for $\mathrm{CO}_{2}$ removal for the desired time depending on the targeted length of the hydrophobic block. The final self-assembled product was dialyzed against DMSO using Spectra/Por 6 Dialysis Tubing, $3.5 \mathrm{kDa}$ MWCO, $45 \mathrm{~mm}$ flatwidth. To isolate the polymer for characterization, an aliquot was precipitated in diethyl ether, washed three times with diethyl ether, dried, and analyzed using ${ }^{1} \mathrm{H}$-nuclear magnetic resonance (NMR) for DP calculation and gel permeation chromatography (GPC) for MW estimation. The different assemblies were transferred to aqueous solutions by means of dialysis against water using Spectra/Por 6 Dialysis Tubing, $3.5 \mathrm{kDa}$ MWCO, $45 \mathrm{~mm}$ flat-width. PEO-PPhe polymers were obtained following the same procedure.

${ }^{1} \mathrm{H}$-Nuclear Magnetic Resonance (NMR). NMR spectra were recorded at $27{ }^{\circ} \mathrm{C}(300 \mathrm{~K})$ on a Bruker Avance III 600 spectrometer. Data were processed with the software Mestrenova 9.0. Samples were prepared at a $10 \mathrm{mg} / \mathrm{mL}$ concentration in deuterated trifluoroacetic acid (TFA-d).

Gel Permeation Chromatography (GPC). GPC measurements were performed in aqueous media due to the insolubility of PEOPMet chains in any organic solvent suitable for GPC in organics. For GPC measurements in aqueous media, the polymers were subjected to a previous oxidation step to poly(ethylene oxide)- $b$-poly( $(\mathrm{L}-$ methionine sulfoxide) using 1.1 equiv of $\mathrm{H}_{2} \mathrm{O}_{2}$ in methanol, at room temperature and under vigorous stirring for $24 \mathrm{~h}^{40}$ The oxidized polymers were subjected to dialysis (MWCO $35 \mathrm{kDa}$ ) and isolated by lyophilization. GPC measurements in aqueous media containing $0.1 \mathrm{M}$ of $\mathrm{NaNO}_{3}$ as an additive were performed in an AF2000 system from Postnova Analytics (Landsberg, Germany). The system was configured to work on GPC mode with an isocratic pump (PN1130) and an autosampler (PN5300). As detectors, we used a refractive index (RI, PN3150), 21 angle-multiangle light scattering
(MALS, PN3621), and ultraviolet-visible (UV-vis) (PN3211) detectors. A working flow rate of $0.7 \mathrm{~mL} / \mathrm{min}$ at $25{ }^{\circ} \mathrm{C}$ was employed with one Novema Max 100A Column equipped with a Novema Max Guard Column (PSS Polymer, Germany). Refractive index and multiangle light scattering were used for detection and molecular weight determination. Calibration of both RI and MALS detectors was achieved with well-defined Pullulan $(50 \mathrm{kDa})$ and validation with poly(ethylene glycol) $(21 \mathrm{kDa})$ standards. A polymer solution $(50 \mu \mathrm{L}$, $3 \mathrm{mg} / \mathrm{mL}$ ) was injected each time.

Transmission Electron Microscopy (STEM). The morphology and size of the structures were imaged using a JEOL 100CX (Japan) microscope $(100 \mathrm{kV})$ equipped with a Gatan camera ERLANGSHEN ES500W Model 782. We decided to use a low-voltage microscope due to the beam sensitivity of polypeptide samples. Prior to TEM imaging, the samples were stained with $0.5 \%(\mathrm{w} / \mathrm{v})$ phosphotungstic acid (PTA) (Sigma-Aldrich, Dorset, U.K. ${ }^{41}$ ). Briefly, carbon-coated 400 mesh copper grids (Agar Scientific, Essex, U.K.), were glowdischarged for $40 \mathrm{~s}$ to render them hydrophilic. Next, $5 \mu \mathrm{L}$ of the sample dispersions, which were diluted fivefold to a concentration of $0.5 \mathrm{mg} / \mathrm{mL}$, were deposited on the grids for $1 \mathrm{~min}$, followed by removal of the excess of sample using a filter paper. The specimens were positively stained by immersion into a $0.5 \%$ PTA solution for $4 \mathrm{~s}$. The grids were blotted again and dried under vacuum for $1 \mathrm{~min}$.

Conventional Scanning Transmission Electron Microscopy (STEM). Conventional STEM imaging was performed using a JEOL JEM-2200FS TEM equipped with a field emission gun (FEG) at 200 $\mathrm{kV}$, and an in-column Omega $(\Omega)$ filter. STEM mode imaging was performed using a HAADF detector and a dwelling time of $13 \mu \mathrm{s}$.

Liquid Scanning Transmission Electron Microscopy (Liquid STEM). The liquid holder Ocean from DENS solutions was used for imaging the dynamics of the vesicle and gel specimens. The liquid samples were encased into two silicon nitride $\left(\mathrm{Si}_{x} \mathrm{~N}_{y}\right)$ chips. These chips had a $50 \mathrm{~nm}$ thick $\mathrm{Si}_{x} \mathrm{~N}_{y}$ electron-transparent window of dimensions $10 \mu \mathrm{m} \times 200 \mu \mathrm{m}$. One of these chips had a $200 \mathrm{~nm}$ spacer to keep the liquid thickness constant in the experiments. The chips were cleaned in HPLC-grade acetone followed by isopropanol for 5 min each, to remove their protective layer made of poly(methyl methacrylate) (PMMA). Afterward, the chips were plasma-cleaned for $13 \mathrm{~min}$ to increase their hydrophilicity. Specimen $(1.5 \mu \mathrm{L})$, vesicles $\left(\mathrm{PEO}_{125}-\mathrm{PMET}_{80}\right)$, and worm micelle-based gel-like structures $\left(\mathrm{PEO}_{125}-\mathrm{PMET}_{40}\right)$, at $2.5 \mathrm{mg} / \mathrm{mL}$ in water, were deposited on the previously prepared $200 \mathrm{~nm}$ spacer chip. The drop-cast specimen was enclosed by the spacer-less chip; right after, the liquid holder was closed, to seal the liquid chamber. The sample solution $(300 \mu \mathrm{L}, 2.5$ $\mathrm{mg} / \mathrm{mL}$ ) was flushed in the liquid holder at $20 \mu \mathrm{L} / \mathrm{min}$ with a syringe pump to ensure the liquid cell inlet and outlet pipes were filled out with solution. The liquid holder was introduced in the transmission electron microscope (TEM). Imaging was performed under static conditions, i.e., not in flow.

The soft polymer nanoparticles were imaged and recorded using a JEOL JEM-2200FS transmission electron microscope (TEM) equipped with an Omega $(\Omega)$ filter and a field emission gun (FEG) at $200 \mathrm{kV}$. Imaging was performed in STEM mode HAADF to control the amount of energy induced to the specimen. The imaging was performed with a built-in Hamamatsu camera. The videos were recorded with the MOVAVI screen recorder software (version 9.5).

The images recorded via liquid scanning transmission electron microscopy (liquid TEM) were corrupted by noise, which significantly reduced the quality of the images, complicating any further analysis. Therefore, the Noise2Void (N2V) machine learningbased approach was adopted to overcome this problem. ${ }^{42}$ Unlike the conventional machine learning-based approach, N2V reduces the noise of an image without any need of the corresponding noiseless image. This requirement makes $\mathrm{N} 2 \mathrm{~V}$ perfect to process LP EM images, as noiseless images in liquid TEM are impossible to record. However, N2V requires a big dataset (also known as training set) to fulfill its task, a common requirement to machine learning-based approaches. Conventionally, the training set has to contain thousands of hundreds of images recorded with the same imaging settings, to produce high-quality estimation of the noise distribution. Unfortu- 

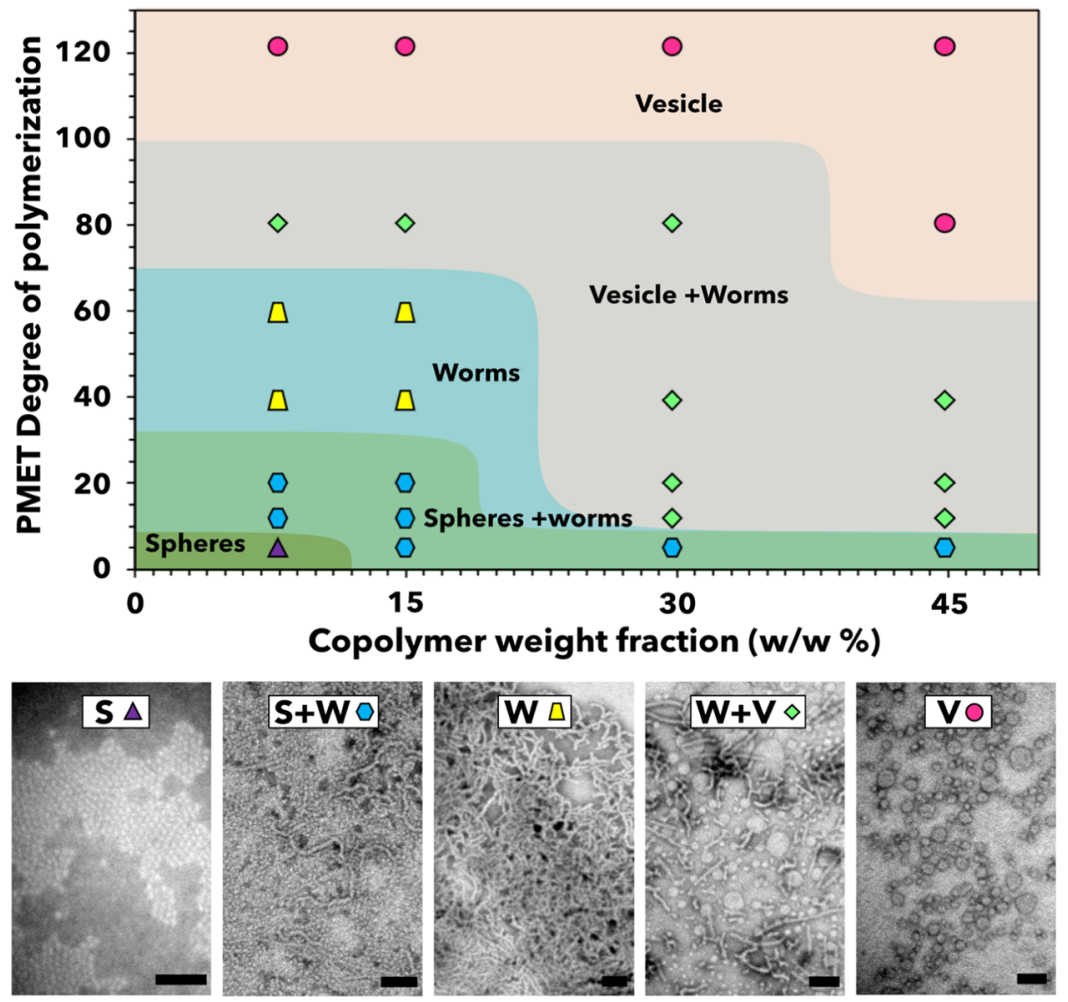

Figure 2. Phase diagram of polypeptide nanoparticle morphologies. Morphological phase diagram (top graph) showing the $\mathrm{PEO}_{125}-\mathrm{PMET}_{x}$ nanoparticle morphologies in aqueous dispersion after solvent displacement by dialysis, for polymerization reactions conducted under various solid contents (expressed in $\mathrm{w} / \mathrm{w} \%$ ) and degrees of polymerization (DP) of the polymethionine (PMET) block. Symbol legend: purple triangle = spheres (i.e., spherical micelles) $(S)$; blue hexagon $=$ spheres + worms (i.e., wormlike micelles) $(S+W)$; yellow trapeze $=$ worms $(\mathrm{W})$; green diamond $=$ worms + vesicles $(\mathrm{W}+\mathrm{V})$; pink circle $=$ vesicles $(\mathrm{V})$. The TEM micrographs of stained $\mathrm{PEO}_{125}-\mathrm{PMET}_{x}$ structures in aqueous dispersion $(0.5 \mathrm{mg} / \mathrm{mL})$ after solvent displacement by dialysis show examples of the relative structures. Scale bar $=100 \mathrm{~nm}$.

nately, in our case, only single videos (image sequence) recorded at different imaging conditions were available. Therefore, the training set was created by sampling the image sequence every two frames, in order not to bias the training process of the $\mathrm{N} 2 \mathrm{~V}$. The remaining half of the image sequence has been processed and used to derive the presented results.

To train the N2V, 3050 frames were selected, extracting 128 different nonoverlapping squared patches (i.e., portions of the pixels of the image) of $64 \times 64$ pixels from each of them. Moreover, N2V was trained for 100 epochs, with a batch size of 128 . These values produced the best results in a short processing time. Results of this noise reduction procedure are displayed in Figure S12.

Oxidation Kinetics. For oxidation kinetics, samples of either vesicles or worms were prepared at $1 \mathrm{mg} / \mathrm{mL}$. Samples were then incubated at $37{ }^{\circ} \mathrm{C}$ with different $\mathrm{H}_{2} \mathrm{O}_{2}$ concentrations ranging from 0.1 to $10 \mathrm{mM}$ for $24 \mathrm{~h}$. Absorbance measurements at $450 \mathrm{~nm}$ were performed at each time point using a UV-2401 PC Shimadzu spectrophotometer (Japan) at room temperature, a scan speed of 40 $\mathrm{nm} / \mathrm{min}$, an integration time of $2 \mathrm{~s}$, and a bandwidth of $1 \mathrm{~nm}$. Due to their nanometric size, the nanoparticles are expected to scatter light at the incident wavelength, and therefore, the detected signal can be considered as an indication of the turbidity of the samples.

Circular Dichroism (CD). CD spectroscopy was performed with a J-715 CD spectrometer (JASCO Corporation, Japan) using a Peltier thermostated cell holder (PTC-423, JASCO Corporation) with a recirculating cooler (JULABO F250, JASCO Corporation). Nitrogen flow $(\sim 2.7 \mathrm{~L} / \mathrm{min})$ was led through the spectrometer and controlled with a nitrogen flow monitor (Afriso Euro-Index). The samples were measured in double-distilled water $\left(\right.$ Milli- $\left.\mathrm{Q}_{2} \mathrm{O}\right)$ at $1 \mathrm{mg} / \mathrm{mL}$. The samples were measured repeatedly $(n=3)$ in a quartz cuvette with $d=$ $0.1 \mathrm{~mm}$. Spectra were recorded at $25{ }^{\circ} \mathrm{C}$ from 300 to $180 \mathrm{~nm}$ using a data pitch, a bandwidth of $0.2 \mathrm{~nm}$, and a scan speed of $50 \mathrm{~nm} / \mathrm{min}$.
The final spectra were produced by combining 10 individual spectra and then expressed in molar ellipticity.

\section{RESULTS AND DISCUSSION}

Polypeptide-based block copolymers were synthesized using a variation of the ROP of NCA method based on the synthesis of urethane derivatives of amino acids, as more stable and less moisture-sensitive monomers than NCA monomers. ${ }^{43}$ Such active urethane derivatives are known to be susceptible to cyclization to form NCAs under mild temperature conditions, which can further polymerize depending on the solvent nature. $^{44,45}$ Specifically, hydrophobic blocks of the ROSsensitive activated urethane of methionine were polymerized at $60{ }^{\circ} \mathrm{C}$ in the polar aprotic solvent dimethyl sulfoxide (DMSO) using the hydrophilic block methoxy-poly(ethylene glycol)amine $\left(\mathrm{mPEO}_{125}-\mathrm{NH}_{2}\right)$ as both macroinitiator and stabilizer block (Figure 1a). Although DMSO was a good solvent for the chosen monomer, the reaction mixture became turbid as the reaction progressed, suggesting that the polypeptide was not soluble. The growing solvent-phobic chain established the condition for self-assembly, and the final structures were controlled by the polypeptide degree of polymerization as well as by the solid content in the reaction mixture, the latter defined as the copolymer weight fraction at the end of the reaction, as typically done in the PISA literature (Figure 1c). We tested the strength of our methodology for producing different nanoparticle morphologies by controlling the degree of polymerization (DP) of the methionine block from 5 to 120 units and by varying the total solid content from 8 to $45 \%$ (Figure 1c). 
Due to the high insolubility of the large polymers obtained (i.e., DPs above 40 units) in the common solvents for GPC analysis, the molecular mass and polydispersity $(\boxplus)$ of the generated block copolymers were estimated by gel permeation chromatography (GPC) under aqueous conditions by previously oxidizing the methionine block selectively into methionine sulfoxide (see the Experimental Section (Figure S1a and Table S1)). All of the resulting block copolymers conforming the assembled nanostructures were characterized by nuclear magnetic resonance (NMR) in deuterated trifluoroacetic acid (TFA-d) after polymer isolation (Figure S1b). We obtained the expected molecular masses according to the feed monomer/initiator ratios (by NMR and GPC) and estimated that polydispersity, $Ð$, was below 1.3 (Table S1), except for $\mathrm{PEO}_{125}-\mathrm{PMET}_{120}$, which was close to 1.5. Especially in the case of smaller polymers, the GPC trace shows a small overlap of the copolymer and the initiator (PEO) traces (Figure S1), which might be explained by the presence of impurities, unreacted monomer, or a small fraction of homopolymers resulting from residual unwanted reactions. The reaction was completed in $6 \mathrm{~h}$ for the longest hydrophobic block of $\mathrm{PEO}_{125^{-}}$ $\mathrm{PMET}_{120}$ and a solid content of $8 \% \mathrm{w} / \mathrm{w}$, as demonstrated by negligible changes in composition according to NMR after that time (Figure S2). There was also a nonsignificant change of nanoparticle size measured by dynamic light scattering (DLS) after the $6 \mathrm{~h}$, indicating reaction completion (Figure S2). Increasing the initial monomer concentration resulted in increased viscosities, which led to longer reaction times (Table S2). We obtained defined particles with solid contents as high as $45 \%$, in contrast to the previously reported investigation, ${ }^{25,26}$ demonstrating the strong potential of our method for large-scale production. The polypeptide polymerization and concomitant self-assembly were performed in DMSO. The reaction mixtures were imaged by transmission electron microscopy (TEM), and a variety of assemblies such as micelles, worms, and vesicles were displayed depending on the reaction conditions, and resulting degree of polymerization as well as solid content (Figure S3). Most notably, the generated morphologies were preserved without visible structural changes after transfer to aqueous media (Figures 2, S4, and S5) displacing DMSO via dialysis (MW cutoff $=3.5 \mathrm{kDa}$ ). Such a structural continuity is critical in biological applications, where an aqueous environment is usually preferred. We observed the additional formation of larger aggregates that led to partial sedimentation after solvent displacement (not observed in DMSO), only in samples with the highest concentration ( $45 \%$ solid content). The sedimentation in these particular cases was reduced by diluting the sample prior to solvent displacement.

We then sought to investigate the impact of the degree of polymerization (as the hydrophobic block length) and the copolymer concentration (i.e., solid concentration) on the morphology of the self-assembled nanostructures. Extensive TEM analysis allowed us to generate a phase diagram for PMET degree of polymerization vs solid content, as shown in Figure 2. Only for the smallest targeted DP yielding $\mathrm{PEO}_{125^{-}}$ $\mathrm{PMET}_{5}$, and a solid content of $8 \%$ we observed small spherical aggregates with diameter around 10-15 $\mathrm{nm}$. Increasing the solid content and keeping this particular hydrophobic length fixed at five units of methionine progressively led to a mixture of spherical and wormlike micelles of thickness ca. $20 \mathrm{~nm}$. Further increases of the hydrophobic block length from 5 to 10-20 methionine units, at the lowest concentration tested of
$8 \%$ solid content, resulted in a mixture of mostly linear wormlike micelles and some remaining spherical micelles. A sole population of wormlike micelles was obtained at DPs of 40-60 when keeping the solid content at $8 \%$. Higher DPs produced a mixture of worms and intermediate structures including branched worms, partially coalesced worms, nascent bilayers. ${ }^{15}$ Finally, a vesicle phase was observed with diameters ranging between 100 and $200 \mathrm{~nm}$, with $\mathrm{PEO}_{125}-\mathrm{PMET}_{120}$, at $8 \%$ solid content. A similar trend was observed at $15 \%$ solid content (Figures S3 and S4) when increasing the methionine degree of polymerization from 5 to 120 . Wormlike micelles and vesicle-enriched samples became more predominant for the highest solid contents, i.e., 30 or $45 \%$, above 10 methionine units in both cases (Figures 2 and S6). Remarkably, batch-tobatch reproducibility was consistent between different polymerization batches of the one-pot method $(n=2-3$ depending on the sample) in terms of polymer characterization and obtained nanostructures. It is worth noticing that wormlike micelles obtained for $\mathrm{PEO}_{125}-\mathrm{PMET}_{40}$ further assembled into organogels during polymerization, whose formation was highly dependent on the solid content (Figure S7). Most importantly, like for any of the other assemblies, also the gels were preserved upon dialysis and replacement of DMSO with water (Figures S7 and S8). Both types of gels, i.e., organo- and hydrogels, were self-standing and stayed at the bottom of the vials when these were turned upside down, as shown in Figures S7 and S8. The gel-like behavior of $\mathrm{PEO}_{125}$ $\mathrm{PMET}_{40}$ was confirmed by a larger value of storage modulus, $G^{\prime}$ (connected to the storage of elastic energy of the sample and, thus, to its solidlike behavior), compared to the loss modulus, $G^{\prime \prime}$ (related to the dissipation of viscous energy under shear, i.e., to a liquid-like behavior) when the sample was subjected to a small oscillatory strain over time, as seen in Figure S9a. The values of $G^{\prime}(2890 \pm 50 \mathrm{~Pa})$ and $G^{\prime \prime}(321 \pm$ $18 \mathrm{~Pa})$ were comparable to those of soft tissues in the liver or the brain. ${ }^{46}$ However, the gels could also be injected using a syringe, as shown in Supporting Information Video S1. To mimic injection in oscillatory strain tests, we applied a strong shear rate $\left(500 \mathrm{~s}^{-1}\right)$ for a short period of time $(60 \mathrm{~s})$, which led to a sharp decrease of both $G^{\prime}$ and $G^{\prime \prime}$, with $G^{\prime \prime}$ values very close (almost coincident) to those of $G^{\prime}$. Indeed, when $G^{\prime \prime}$ > $G^{\prime}$, the internal gel network is lost, i.e., shear thinning behavior, which enables injection. ${ }^{47}$ The shear thinning behavior of the polypeptide was more pronounced than for a 15 wt \%. Pluronic F127 gel under the same shear conditions (at $37^{\circ} \mathrm{C}$ ), which is well known for its thermosensitive response and injectability ${ }^{48}$ (Figure S9b). Significantly, the sample gradually recovered consistency after time $\left(G^{\prime}>G^{\prime \prime}\right)$ once the strong shear rate was stopped, indicating a self-healing behavior.

The phase diagram in Figure 2 is dominated by cylindrical micellar and vesicular arrangements. While the latter might be consistent with the hydrophobic/hydrophilic ratios of the copolymer, the extent of formation of the former is surprisingly wide. As the hydrophobic segment increases in mass and size, one should expect a natural evolution from spherical micelles to worms to vesicles. However, we observed spherical arrangements only at very short PMET chains and at a low concentration. A potential explanation for such discrepancy for amphiphilic polypeptides can be attributed to the peptide secondary structure. ${ }^{38}$ Thus, we assessed the secondary structure for the different assemblies using circular dichroism (CD) spectroscopy. The CD spectra collected for all of the different PMET degrees of polymerization are shown in Figure 

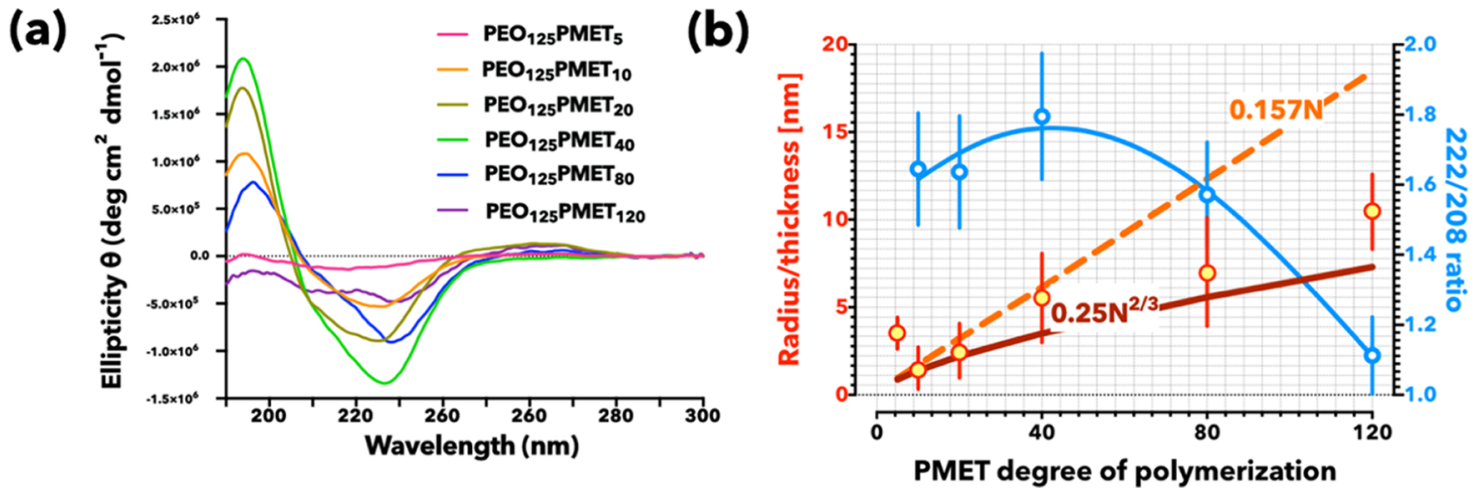

Figure 3. Secondary structure effect on self-assembly. (a) Circular dichroism spectra in water as a function of the polymethionine (PMET) degree of polymerization at $15 \%$ solid content. (b) Scaling analysis between the micelle radius or the vesicle membrane thickness (measured from TEM images) as a function of the PMET degree of polymerization (left $y$-axis, red, yellow-filled circles) plotted alongside two scaling laws: supersegregated copolymers $\left(d=0.25 N^{2 / 3}\right)$ and assuming a perfect $\alpha$-helix conformation $(d=0.157 N)$. The ratio between the two $\alpha$-helix characteristic peaks at wavelengths 222 and $208 \mathrm{~nm}$ vs degree of polymerization is also plotted (right $y$-axis, blue circles).

3a. With the exception of $\mathrm{PEO}_{125}-\mathrm{PMET}_{5}$, and in agreement with the reported propensity of methionine to form helix-like structures, $^{49}$ all spectra showed evidence of $\alpha$-helix conformation with the typical positive peak for wavelengths lower than $190 \mathrm{~nm}$ and negative peaks between 190 and $260 \mathrm{~nm}$. These peaks were particularly intense for PMET degrees of polymerization between 10 and 80 , showing a nonmonotonic trend with the most negative value obtained for $\mathrm{PMET}_{40}$. Indeed, if we plot the ratio between the two $\alpha$-helix characteristic peaks at wavelengths 222 and $208 \mathrm{~nm}$, respectively (Figure $3 \mathrm{~b}$ ), we see that this is about $1.6-1.8$ for $\mathrm{DP}=10,20,40$, and 80 and it decreases to almost 1 for $\mathrm{PMET}_{120}$. The largest polypeptide synthesized in this study also shows the least intense peaks suggesting a mixture of helix and coil conformations. The rest of the polypeptides show more helical arrangements (estimated by a decreased peak intensity at $222 \mathrm{~nm}$ ), and their higher $222 / 208$ ratio suggests interaction between the helices typical of coiled-coil complex formation. ${ }^{50}$ The ellipticity ratio 222/208 is plotted in Figure $3 \mathrm{~b}$ alongside the micelle radius and the vesicle membrane thickness as a function of the PMET degree of polymerization. Both radius and thickness were measured from TEM images (see the Experimental Section for details) and no evident scaling law with the polymerization degree was observed. If we assumed a perfect $\alpha$-helix conformation, where each methionine unit corresponds to a helix with $a=0.157 \mathrm{~nm}$, then the PMET end-to-end distance $(d)$ would scale linearly with the degree of polymerization $(N)$ with $d=a N$, while if we assumed a typical supersegregated regime for amphiphilic copolymers, then the expected scaling would be $d=0.25 \mathrm{~N}^{2 / 3}$ with 0.25 being the monomer length. ${ }^{51}$ Both trends are plotted in Figure $3 \mathrm{~b}$ revealing that the PMET scaling falls within the two, indicating a mixed behavior, with the secondary structure of short PMET more in line with $\alpha$-helix conformations, while longer polypeptides showed a mixed conformation with random coils.

To gain additional information on the secondary structure of the wormlike micelles, the hydrogels formed by a network of these were imaged by scanning transmission electron microscopy (STEM). The high-angle annular dark-field imaging mode (HAADF) in STEM provides enhanced image contrast and reveals spatial features in certain morphologies that are not easily observed in bright/dark-field TEM. STEM images in dark-field mode revealed a morphology correspond- ing to rather twisted structures in helix-like patterns instead of smooth straight worms as seen in Figure 4a. We also analyzed the same gels formed in the $\mathrm{PEO}_{125}-\mathrm{PMET}_{40}$ sample using liquid-phase scanning electron microscopy (LSTEM), and here, the same structure was observed in water, suggesting that the twisted arrangement was not an artifact from the water removal in dry-state imaging (Figure 4b). Both ultrastructure analyses suggested that the PMET chains folded into $\alpha$-helices and these assembled forming a staggered coiled-coil configuration as shown in Figure 4c. Such an arrangement only allows one direction for growth, and hence, the copolymers form wormlike micelles even though the hydrophilic/hydrophobic ratio would be more optimal for spherical packing. Remarkably, the same sample can however show both wormlike micellar and membrane vesicular morphologies (as shown in Figure 4d), and such this eclectic nature can be easily explained by invoking the staggering of the helices during the formation of the coiled-coil complexes. As we propose in Figure $4 \mathrm{e}$, the parallel arrangement will only allow for membrane packing. Finally, for longer hydrophobic blocks such as $\mathrm{PMET}_{80}$ or $\mathrm{PMET}_{120}$, their ability to form vesicles is demonstrated in liquid-phase STEM micrograph in Figure 4f, and here, we propose a mixed helix and random coil configuration as shown in Figure $4 \mathrm{~g}$ in agreement with both $\mathrm{CD}$ and scaling analyses.

Overall, the novelty of our strategy lies in the production of a variety of morphologies obtained mostly by tuning the targeted length of the hydrophobic blocks, which is controlled by the synthetic procedure. Indeed, NCA polymerization, polymer isolation, and postpolymerization assembly, apart from involving multiple additional steps of purification, did not allow us to obtain the same morphologies. For instance, solvent-switch methods that rely on the use of water-miscible solvents to first dissolve the polymer, which is then transferred to an aqueous solution for self-assembly, could not be used for the whole range of hydrophobic lengths synthesized here. This is the case of large $\mathrm{PEO}_{125}-\mathrm{PMET}_{80-120}$ polymers rendering vesicular morphologies, which turned out to be insoluble in any known water-miscible solvent. In the particular case of $\mathrm{PEO}_{125}-\mathrm{PMET}_{40}$, solvent-switch methods did not yield wormlike micellar hydrogels in any of our attempts. We have also employed time-consuming film-hydration protocols using water-immiscible solvents such as chloroform to dissolve the polymer. Using this method, a minimum of 6-7 weeks was 


\section{(a)}
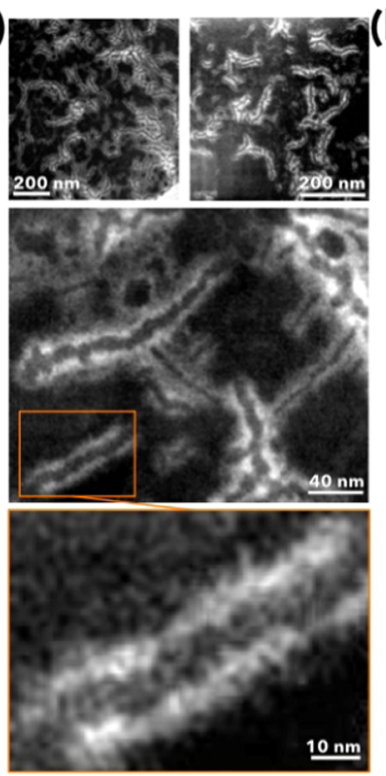

b)

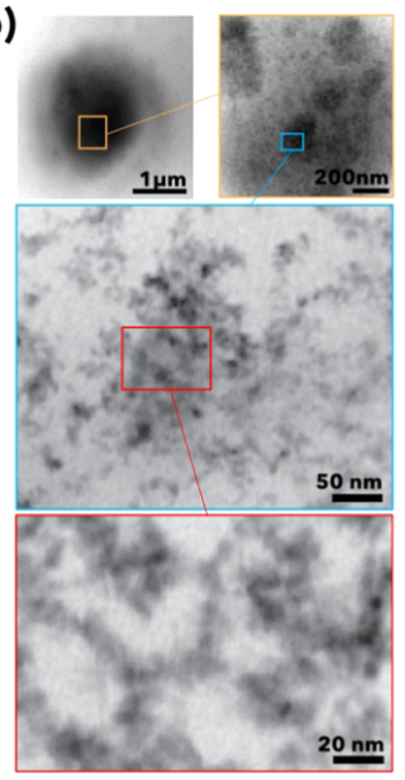

(c)

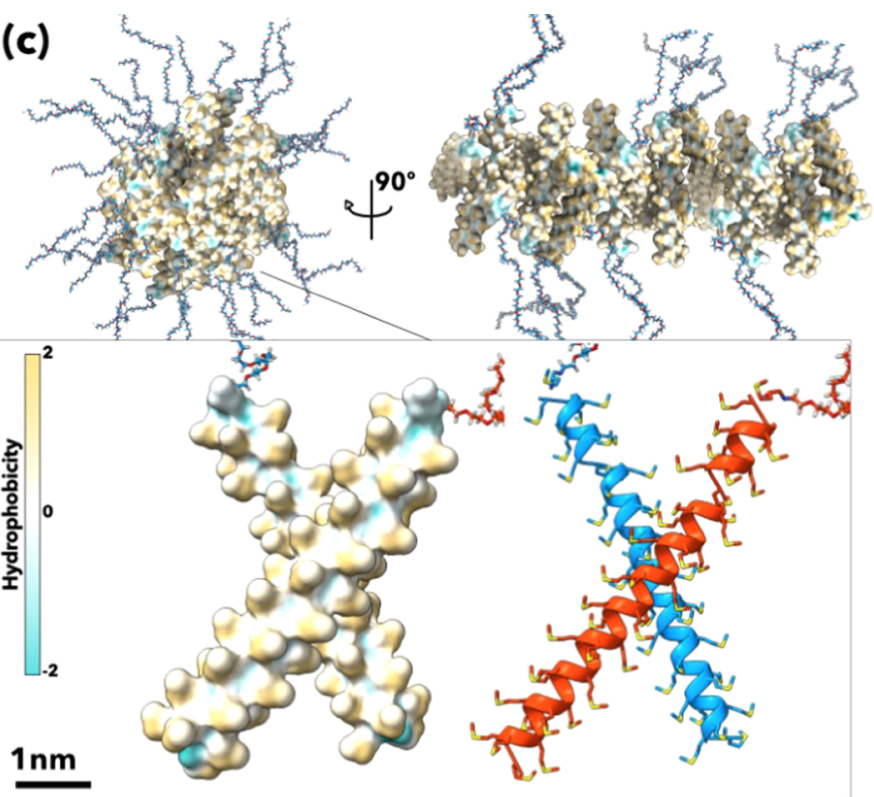

(d)

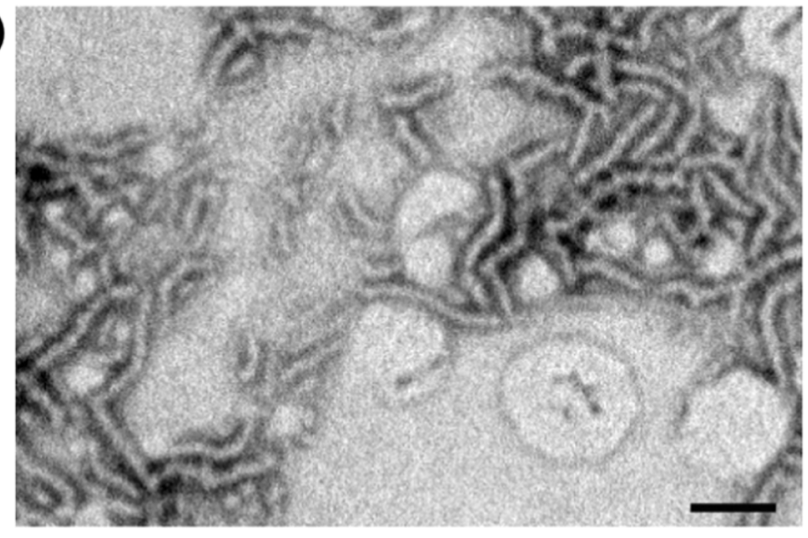

(f)

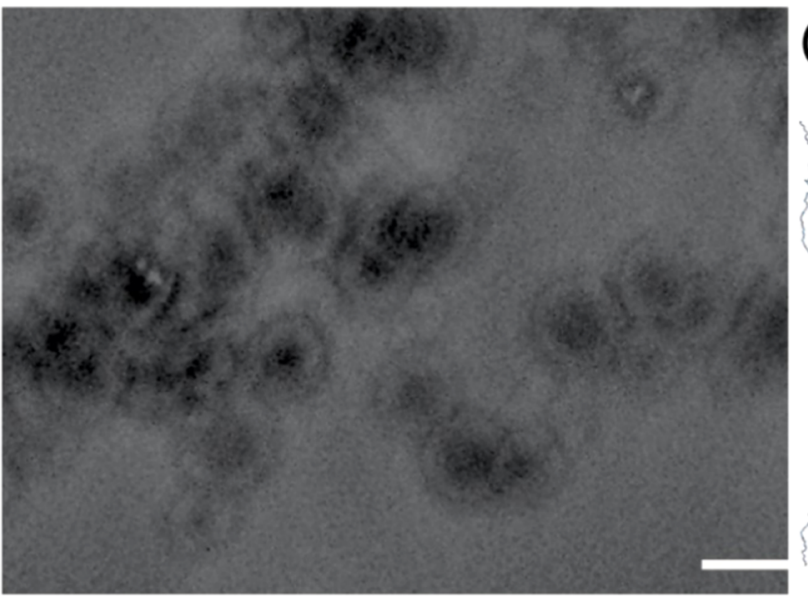

(e)
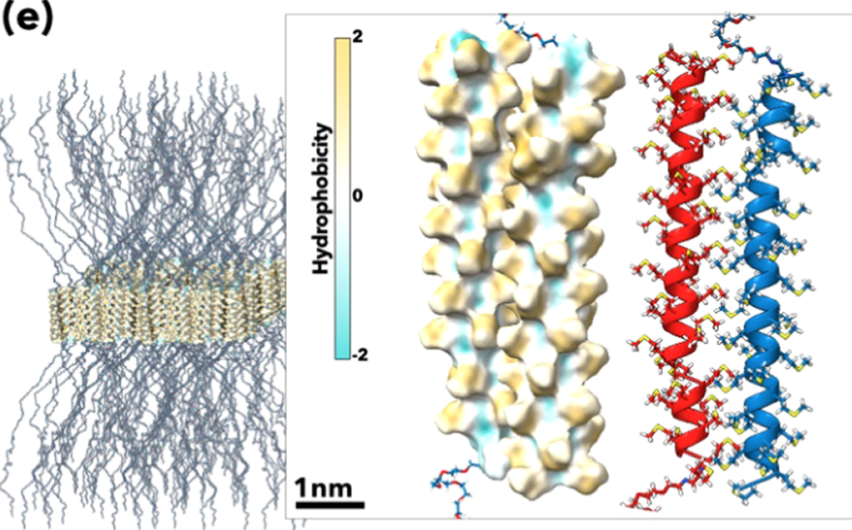

(g)
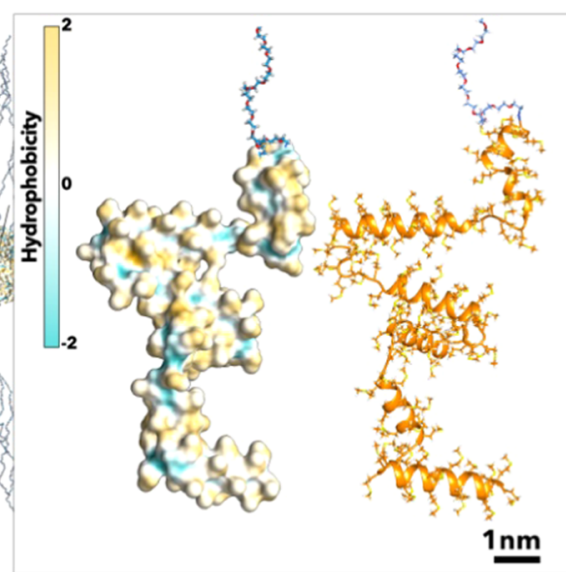

Figure 4. Supramolecular architecture. High-angle annular dark-field scanning transmission electron microscopy imaging (HAADF-STEM) (a) and liquid-phase scanning transmission electron microscopy (LSTEM) imaging (b) of the of $\mathrm{PEO}_{125}-\mathrm{PMET}_{40}$ wormlike micellar gels at different magnifications. Model of the polypeptide assembly showing the $\mathrm{PEO}_{125}-\mathrm{PMET}_{40}$ helices staggered (c). Transmission electron microscopy (d) of $\mathrm{PEO}_{125}-\mathrm{PMET}_{40}$ wormlike micelles coexisting with vesicles (scale bar $=100 \mathrm{~nm}$ ). The membrane arrangement is possible with parallel coiled-coil helices (e). Liquid-phase STEM of $\mathrm{PEO}_{125}-\mathrm{PMET}_{120}$ vesicles (f) and our proposed model of mixed coil and helix conformation ( $\mathrm{g}$ ). Note that the peptide secondary structures are represented showing both the molecule electron density colored as a function of its hydrophobicity as well as with the relevant helices depicted as a cartoon ribbon. Dwelling time for (b): $13 \mathrm{~s} . \mathrm{e}^{-}$doses for (b): $0.008 \mathrm{e}^{-} / \mathrm{A}^{2}$ for top left, $0.19 \mathrm{e}^{-} / \mathrm{A}^{2}$ for top right, $3.11 \mathrm{e}^{-} / \mathrm{A}^{2}$ for centered, and $19.45 \mathrm{e}^{-} / \mathrm{A}^{2}$ for bottom. Scale bar for $(\mathrm{f}): 100 \mathrm{~nm}$. Dwelling time for (f): $3 \mathrm{~s}$. $\mathrm{e}^{-}$doses for $(\mathrm{f}): 47.59 \mathrm{e}^{-} / \mathrm{A}^{2}$.

needed to obtain much more polydisperse spherical vesicles requiring additional steps of size-exclusion chromatography purification and with yields below $40 \%$. Hydrogels were not obtained using this technique either. Overall, our strategy of 
(a)

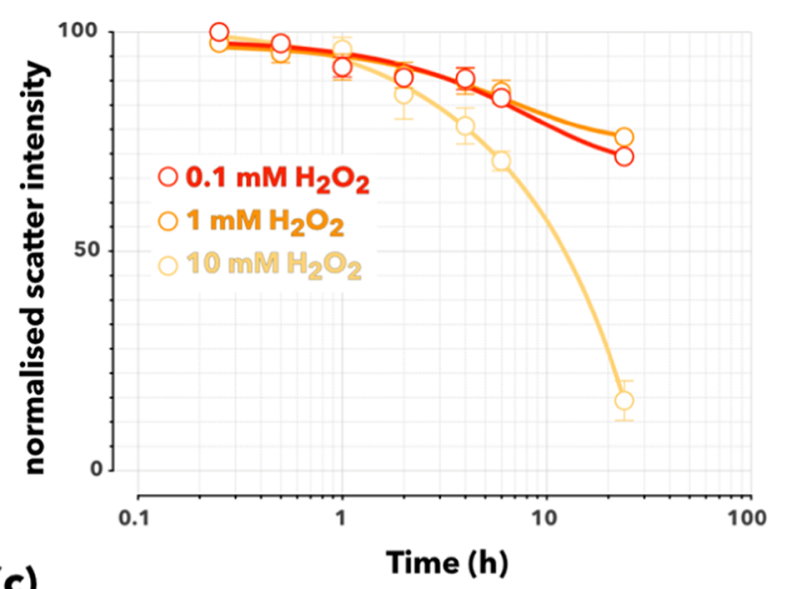

(b)
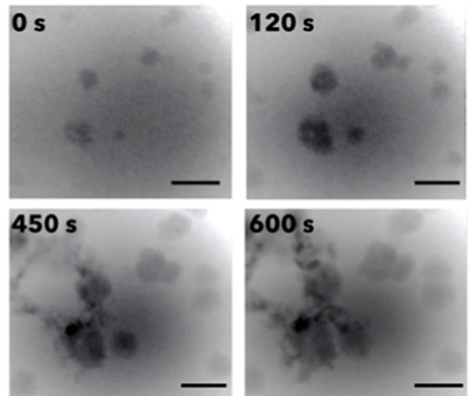

\section{$600 \mathrm{~s}$}
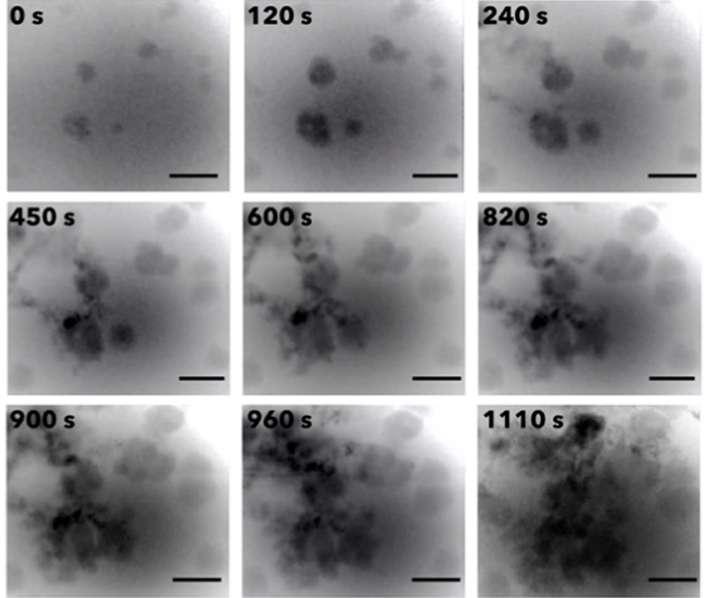

(c)
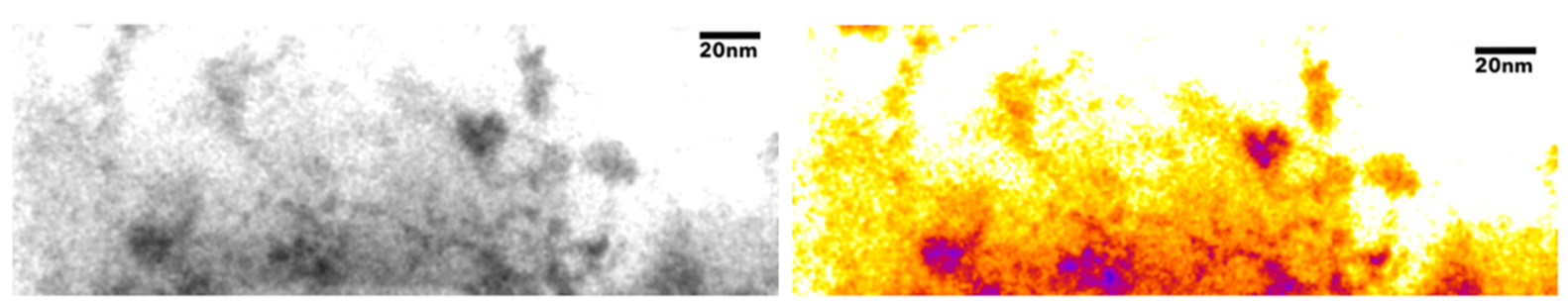

(d)

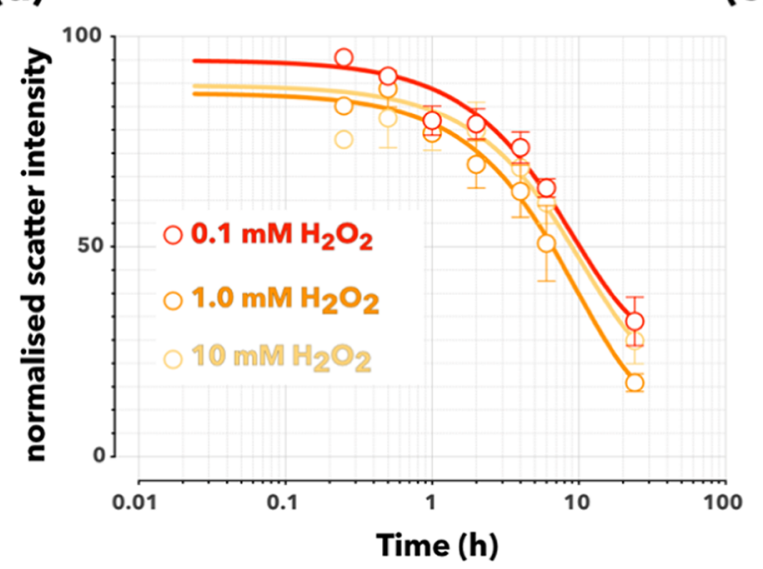

(e)
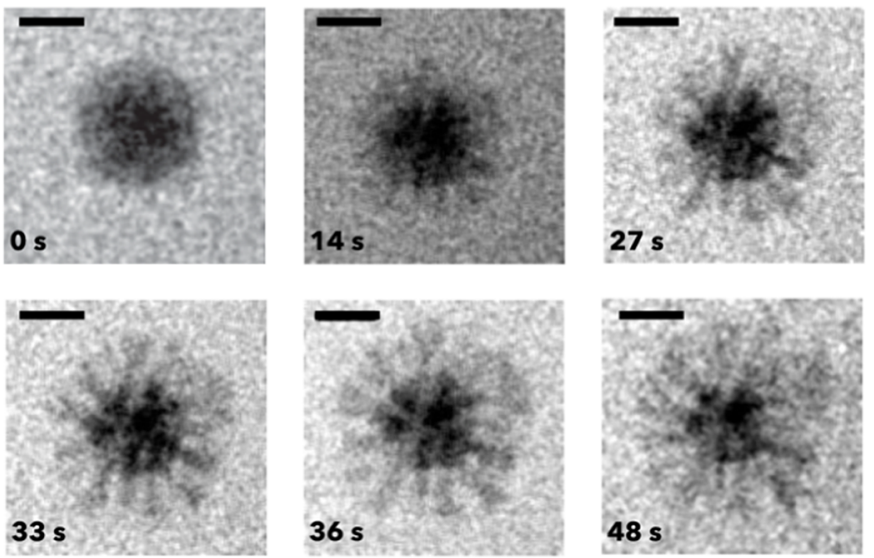

(f)
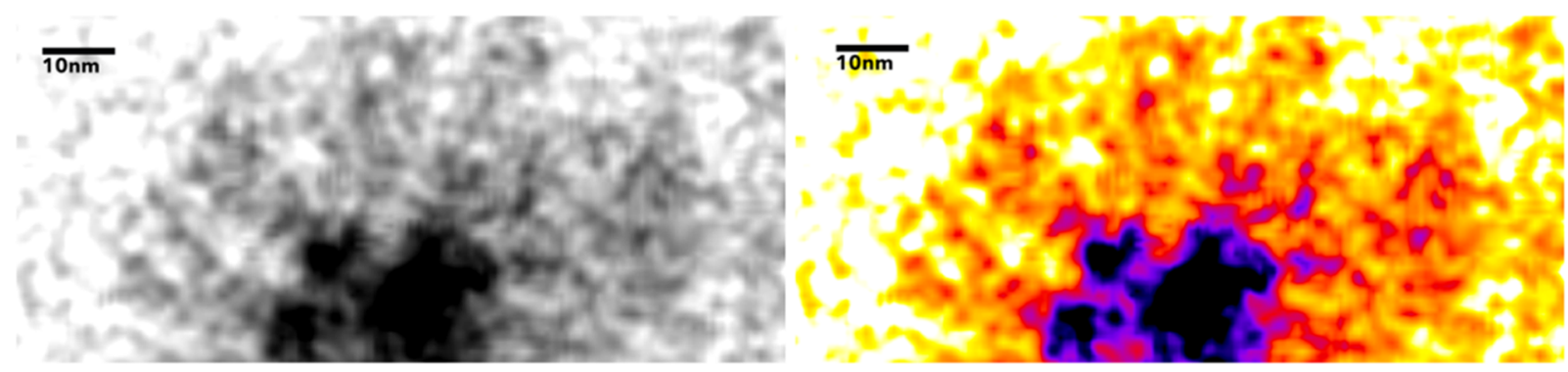

Figure 5. ROS-sensitive disassembly of $\mathrm{PEO}_{125}-\mathrm{PMET}_{x}$ nanostructures. (a) Temporal evolution of the light intensity scattered by $\mathrm{PEO}_{125}$ - $\mathrm{PMET}_{40}$ worms, as a function of time upon the addition of $\mathrm{H}_{2} \mathrm{O}_{2}$ in three different concentrations $(0.1,1$, and $10 \mathrm{mM})$. (b) Time series snapshots of the acquired Video $\mathrm{S} 6$ of $\mathrm{PEO}_{125}-\mathrm{PMET}_{40}$ gel via liquid STEM showing the in situ electron beam-induced degradation occurring progressively in the spherical aggregates located within the gel micron-sized structure. Scale bar $=200 \mathrm{~nm}$. $\mathrm{e}^{-}$dose rate: $33.3 \mathrm{e}^{-} / \mathrm{A}^{2} / \mathrm{s}$, total dose: $40626 \mathrm{e}^{-} / \mathrm{A}^{2}$. Liquid STEM videos provided in the SI. (c) Magnification showing the closely packed worms inside the globular gels. (d) Temporal evolution of the light intensity scattered by vesicles, as a function of time upon the addition of $\mathrm{H}_{2} \mathrm{O}_{2}$ in three different concentrations $(0.1,1$, and $10 \mathrm{mM}$ ). (e) Liquid STEM micrographs showing disassembly of a vesicle in real time by the oxidation of the PMET block as a consequence of electron beam irradiation in the aqueous media. The series starts at $0 \mathrm{~s}$ when the polymer vesicle is intact; after $14 \mathrm{~s}$, the polymer vesicle membrane shows initial changes in the form of spikes protruding out of the membrane; the vesicle disassembly process progresses by growing tentacle-like branches; at $48 \mathrm{~s}$, a final hyperbranched structure with an inhomogeneous spherical core is reached. Dwelling time $=13 \mu \mathrm{s}$. Scale bar $=100 \mathrm{~nm}$. Dose rate: $47.6 \mathrm{e}^{-} / \mathrm{A}^{2} / \mathrm{s}$, total dose: $2284.8 \mathrm{e}^{-} / \mathrm{A}^{2}$. (f) Magnification of the final hyperbranched structure obtained in (e). 
self-assembly during polymerization is governed by coil-coil interactions that occur during polymerization, therefore leading to different morphologies compared to conventional methods, also in high yields. Additionally, the versatility of this strategy has been successfully extended to the PISA of other hydrophobic amino acid, phenylalanine. Figure S10 shows TEM micrographs depicting how different morphologies ranging from micelles ( $\mathrm{DP}=5)$ to wormlike hydrogels (DP $=40)$, up to vesicular hydrogels ( $\mathrm{DP}=80)$, can be obtained by tuning the hydrophobic block (polyphenylalanine) length at a constant polymer concentration of $8 \%$ solid content. The vesicular hydrogel phase formation was previously reported for amphiphilic copolymers at a high solid content. ${ }^{52-54}$ Therefore, we envisage that nanoassemblies based on any hydrophobic amino acid (or amino acid mixtures), soluble in DMSO and insoluble when polymerized, could be potentially obtained following this methodology.

Finally, we investigated the potential stimuli-responsiveness of the assemblies against reactive oxygen species (ROS). For exemplary purposes, we used $\mathrm{H}_{2} \mathrm{O}_{2}$, one of the main species found in the human body, although not the only one. While still controversial due to the variability of results reported in the literature, the absolute $\mathrm{H}_{2} \mathrm{O}_{2}$ concentration in the blood is thought to be in the range of $1-5 \times 10^{-3} \mathrm{mM}$ under healthy conditions and $30-50 \times 10^{-3} \mathrm{mM}$ during chronic inflammation. ${ }^{55}$ However, local $\mathrm{H}_{2} \mathrm{O}_{2}$ concentrations generated by activated macrophages in disease conditions may reach up to $0.01-1 \mathrm{mM}{ }^{56}$ Thus, if properly targeted, this distinctive local redox environment in pathological vs healthy regions offers a great opportunity for disease diagnosis and/or therapy. Methionine possesses a thioether moiety that is well known to undergo oxidation under the exposure to common oxidants (including $\mathrm{H}_{2} \mathrm{O}_{2}$ ), yielding first methionine sulfoxide under mild oxidation, and finally methionine sulfone, under further oxidation. ${ }^{57}$ Both species are hydrophilic, and therefore, the hydrophobic-to-hydrophilic transition of methionine allows for the design of ROS-responsive nanostructures. We designed a series of UV-vis spectrophotometry experiments to assess the sample turbidity as a measure of the scattering effect of the nanoparticles on light upon addition of hydrogen peroxide $\left(\mathrm{H}_{2} \mathrm{O}_{2}\right)$. We found that the wormlike micelle-containing samples remained roughly stable when applying the lowest, and physiologically relevant, $\mathrm{H}_{2} \mathrm{O}_{2}$ concentrations tested of 0.1 and $1 \mathrm{mM}$, displaying only about $20 \%$ decrease in the scattering signal after $24 \mathrm{~h}$ of incubation (Figure 5a). Nevertheless, wormlike micelle samples were found almost completely degraded when subjected to the highest $\mathrm{H}_{2} \mathrm{O}_{2}$ concentration of the study at $10 \mathrm{mM}$. Thus, the degradation rate of the wormlike micelle gels was clearly dependent on the ROS concentration, making these materials promising candidates, for instance, as drug delivery depots in ROSrelated diseases. Our results agree with previous reports by $\mathrm{Xu}$ et al., who showed a slow and sustained ROS-dependent degradation of similar hydrogels at $1 \mathrm{mM} \mathrm{H}_{2} \mathrm{O}_{2}$ over 19 days of incubation, and a fast hydrogel disruption in 3 days at $10 \mathrm{mM}$ $\mathrm{H}_{2} \mathrm{O}_{2} .{ }^{58}$ Reactive radical species have been reported to form in aqueous media upon electron beam irradiation, ${ }^{59,60}$ which allowed us to use liquid EM imaging as a tool to monitor in real time the polymer degradation by a different oxidation process. Liquid STEM (Videos S2-S8) disclosed that the worm-based gels seemed to be compartmentalized into large micron-sized globular structures (Figures $5 \mathrm{~b}$ and S11) with different densities at the core and outer regions, confining closely packed worms in their interior. Upon electron beam irradiation, the degradation displayed as progressive conformational changes due to the change in hydrophobicity of the PMET block. Specifically, these globular aggregates of the gel sample seemed to swell and open up to release wormlike micelles (Figures 5c and S12) in a timescale of ca. $20 \mathrm{~min}$ (Figure 5b). The formerly amphiphilic worms that were packed within the globular structures would have become fully hydrophilic upon oxidation. To maximize contact with the outer solvent, the hydrophilic worms were released from the globules and slowly untangled to be fully exposed to the bulk media. Ultimately, the worm structures changed shape and degraded as seen in Figures 5b,c, S13, and Videos S4 and S6. These observations suggest that the tightly packed network of worms observed in dry TEM/STEM would have been further enclosed within the gel globular structures when the system was in a fully hydrated state. From the contrast offered by the liquid STEM images (Figure 5b,c and Videos S2-S8), it could be hypothesized that the interior of the globular gel structures was filled with a more dense and viscous fluid than the surrounding bulk media. Whether the worms coiled up inside the globular gels in an attempt to minimize contact with the bulk due to their amphiphilic nature or the whole structure simply did not disperse well is unknown. The slow degradation of the wormlike micelle samples could be attributed to the tendency of these structures to form gels.

On the contrary, for vesicles (Figure 5d), the scattering intensity in UV-vis spectrophotometry experiments was reduced from 100 to $0 \%$ after $24 \mathrm{~h}$, suggesting nearly complete degradation of the nanoparticles for all of the $\mathrm{H}_{2} \mathrm{O}_{2}$ concentrations used. About $50 \%$ of the scattered intensity was lost in the vesicle sample after $6 \mathrm{~h}$ of exposure to $\mathrm{H}_{2} \mathrm{O}_{2}$ regardless of its concentration. Further confirmation of nanoparticle disassembly upon oxidation with $\mathrm{H}_{2} \mathrm{O}_{2}$ was obtained by dynamic light scattering techniques, where the lack of a good correlation function for the scattered intensity indicated vesicle disassembly (Figure S14a-c). Moreover, ${ }^{1} \mathrm{H}$ NMR in TFA- $d$ before and after the oxidation process with $\mathrm{H}_{2} \mathrm{O}_{2}(24 \mathrm{~h})$ revealed the complete oxidation of methionine (Figure S14d). These results were in good agreement with previous studies reported by Deming and co-workers in polypeptide-based vesicles bearing a reduction-sensitive poly(methionine sulfoxide) block. ${ }^{38}$ Indeed, Deming et al. explored payload release from vesicles upon membrane disruption due to the enzymatic reduction of methionine sulfoxide into methionine. According to the authors, oxidation produced a conformational change in the inherent secondary structure of the polypeptide block, from $\alpha$-helix in poly(methionine) to a water-soluble disordered conformation in poly(methionine sulfoxide), and finally to a slightly water-soluble form composed of essentially $\alpha$-helix in poly(methionine sulfone). $\mathrm{CD}$ measurements of completely oxidized worms and vesicles can be found in Figure S14e, showing the conformational changes described by Deming et al. We observed that upon oxidation, a sample of $\operatorname{PEO}_{125}-\operatorname{PMET}\left(\mathrm{O}_{x}\right)_{40}$ (worms before oxidation) preserved the $\alpha$-helix conformation, while the coilcoil conformation was lost. In the case of $\mathrm{PEO}_{125}$-PMET$\left(\mathrm{O}_{x}\right)_{120}$ (vesicles before oxidation), the oxidation resulted in a loss of the secondary structure with $\mathrm{CD}$ showing random coil configuration, confirming that high hydrophobic block lengths do not favor $\alpha$-helix configuration. In the present study and in the case of vesicle samples, we did not observe remarkable differences in degradation for all of the $\mathrm{H}_{2} \mathrm{O}_{2}$ concentrations 
tested. When investigated in hydrated state under liquid STEM, the oxidation-sensitive polymer vesicles were observed to progressively change their morphology from membranebound spherical to irregular, hyperbranched structures upon electron beam irradiation (Figures 5e,f and S15). Indeed, the methionine $\rightarrow$ methionine sulfoxide $\rightarrow$ methionine sulfone progressive switch from hydrophobic to hydrophilic generated an escape of the initially hydrophobic block from the vesicle inner membrane to the outside media with the aim of maximizing hydration. This progressive oxidation reaction was monitored in situ as conformational changes, i.e., vesicle degradation, took place in $1 \mathrm{~min}$ (Figure 5e).

Altogether, these results suggest different kinetics of structural change for the two morphologies investigated, i.e., vesicles and wormlike micelles. It seems probable that both transitions, i.e., change of hydrophilicity and conformation, caused a disruption of the structure and further enlargement of the curvature at the hydrophobic-hydrophilic interface, therefore triggering morphological transformations.

\section{CONCLUSIONS}

The preparation of self-assembled polypeptide-based nanoparticles following ring-opening polymerization-induced selfassembly (ROPISA) at relatively high solid content was successfully achieved in a one-pot reaction. The proposed method is based on the ROPISA of low moisture-sensitive urethane derivative NCA precursors with the ability to generate in situ NCAs in polar aprotic solvents such as DMSO, followed by transfer to water via dialysis without the need of an additional self-assembly step. We have applied this method to the hydrophobic and redox stimuli-responsive amino acid methionine in conjunction with hydrophilic poly(ethylene oxide) as macroinitiator and stabilizer block during ROPISA. Additionally, the presented approach has been further extended to the hydrophobic amino acid phenylalanine, proving its versatility. Our strategy allows us to work at high concentrations (up to $45 \%$ solid contents) and aiming for various hydrophobic block lengths (up to 120 units). The control over both parameters allows us to finely tune the system for engineering a wide range of structures from spherical to wormlike micelles and vesicular structures. However, we could not find spherical micelles for methionine block lengths higher than five units and our phase diagram was dominated by cylindrical arrangements. We propose that this is the result of the methionine tendency to form $\alpha$-helices and how these interact with one another. Indeed, we observed the formation of rather twisted structures conforming helix-like patterns instead of smooth linear worms. Remarkably, wormlike micelles further assemble into self-standing gels confined in globular structures, which further expands the spectrum of obtained formulations. Moreover, because of the ROS-responsive methionine block, the nanostructures can be degraded under controlled oxidant conditions, such as exposure to hydrogen peroxide, or induced by an electron beam and monitored in situ when imaged by liquid transmission electron microscopy. The one-pot nature of the reaction, the use of DMSO as reaction solvent (defined as Class 3 solvent with low toxic potential by the European Medicines Agency, EMA and the Food and Drug Administration, FDA), and the easy scalation of dialysis procedures by, i.e., flow dialysis, make our strategy amenable for potential scale-up processes. Finally, the control over the synthesis of defined nanoparticle morphologies using biocompatible, biodegradable, and stimuli-responsive polypeptides can have a myriad of applications in the healthcare sector. We envisage the design of biodegradable wormlike micelle-based amphiphilic hydrogels as biomaterial scaffolds for cellular matrixes, including the option of sustained release of therapeutics. Indeed, the values of the storage and loss moduli were the same order of magnitude as those of soft tissues in the liver or the brain for PEO-PMET 40 hydrogel. Additionally, oxidationsensitive vesicles can be optimized for the encapsulation of key therapeutic agents for pathological conditions involving ROS overproduction, such as stroke, arthritis, and overall, inflammation processes.

\section{ASSOCIATED CONTENT}

\section{Supporting Information}

The Supporting Information is available free of charge at https://pubs.acs.org/doi/10.1021/acs.biomac.1c01039.

Dynamic light scattering (DLS), rheological characterization of $\mathrm{PEO}_{125}-\mathrm{PMET}_{40}$ gels, and additional polymer and nanoparticle characterization and nanoparticle oxidation processes (PDF)

Video illustrating the injectability of $\mathrm{PEO}_{125}-\mathrm{PMET}_{40}$ hydrogels in the laboratory settings (MOV)

Liquid STEM video displaying the worm-like micelles of the oxidation-sensitive polymer $\mathrm{PEO}_{125}-\mathrm{PMET}_{40}$ that are imaged in globular structures (MOV)

Liquid STEM video displaying the worm-like micelles of the oxidation-sensitive polymer $\mathrm{PEO}_{125}-\mathrm{PMET}_{40}$, imaged within the globular structures and their morphological changes due to the radical oxygen species induced by long exposure to the electron beam (MOV)

Liquid STEM video displaying the worm-like micelles of the oxidation-sensitive polymer $\mathrm{PEO}_{125}-\mathrm{PMET}_{40}$, imaged within the globular structures and their morphological changes due to the radical oxygen species induced by long exposure to the electron beam (MOV)

Liquid STEM video displaying the worm-like micelles of the oxidation-sensitive polymer $\mathrm{PEO}_{125}-\mathrm{PMET}_{40}$ that are imaged in globular structures (MOV)

Liquid STEM video displaying the worm-like micelles of the oxidation-sensitive polymer $\mathrm{PEO}_{125}-\mathrm{PMET}_{40}$, imaged within the globular structures and their morphological changes due to the radical oxygen species induced by long exposure to the electron beam (MOV)

Liquid STEM video displaying the worm-like micelles of the oxidation-sensitive polymer $\mathrm{PEO}_{125}-\mathrm{PMET}_{40}$, imaged within the globular structures and their morphological changes due to the radical oxygen species induced by long exposure to the electron beam (MOV)

Liquid STEM video displaying the worm-like micelles of the oxidation-sensitive polymer $\mathrm{PEO}_{125}-\mathrm{PMET}_{40}$ that are imaged at the edge of the globular structures after long time exposure to the beam (MOV)

\section{AUTHOR INFORMATION}

\section{Corresponding Authors}

Aroa Duro-Castano - Department of Chemistry, University College London, London WC1H OAJ, U.K.; Institute for the 
Physics of Living Systems, University College London, London WC1E 6BT, U.K.; 다이.org/0000-0001-7279-854X; Email: aroa.castano@ucl.ac.uk

Giuseppe Battaglia - Department of Chemistry, University College London, London WC1H OAJ, U.K.; Institute for the Physics of Living Systems, University College London, London WC1E 6BT, U.K.; The EPSRC/Jeol Centre for Liquid Phase Electron Microscopy, University College London, London WC1H OAJ, U.K.; Institute for Bioengineering of Catalunya (IBEC), The Barcelona Institute of Science and Technology (BIST), 08028 Barcelona, Spain; Catalan Institution for Research and Advanced Studies (ICREA), 08010 Barcelona, Spain; (1) orcid.org/0000-0003-3349-6770;

Email: g.battaglia@ucl.ac.uk, gbattaglia@ibecbarcelona.eu

\section{Authors}

Laura Rodríguez-Arco - Department of Chemistry, University College London, London WC1H OAJ, U.K.; Institute for the Physics of Living Systems, University College London, London WC1E 6BT, U.K.; Department of Applied Physics, University of Granada, 18071 Granada, Spain; O orcid.org/00000002-5527-7547

Lorena Ruiz-Pérez - Department of Chemistry, University College London, London WC1H OAJ, U.K.; Institute for the Physics of Living Systems, University College London, London WC1E 6BT, U.K.; The EPSRC/Jeol Centre for Liquid Phase Electron Microscopy, University College London, London WC1H 0AJ, U.K.

Cesare De Pace - Department of Chemistry, University College London, London WC1H 0AJ, U.K.; Institute for the Physics of Living Systems, University College London, London WC1E 6BT, U.K.; The EPSRC/Jeol Centre for Liquid Phase Electron Microscopy, University College London, London WC1H 0AJ, U.K.

Gabriele Marchello - Department of Chemistry, University College London, London WC1H 0AJ, U.K.; Institute for the Physics of Living Systems, University College London, London WC1E 6BT, U.K.; The EPSRC/Jeol Centre for Liquid Phase Electron Microscopy, University College London, London WC1H OAJ, U.K.; Institute for Bioengineering of Catalunya (IBEC), The Barcelona Institute of Science and Technology (BIST), 08028 Barcelona, Spain

Carlos Noble-Jesus - Department of Chemistry, University College London, London WC1H OAJ, U.K.; Institute for the Physics of Living Systems, University College London, London WC1E 6BT, U.K.

Complete contact information is available at:

https://pubs.acs.org/10.1021/acs.biomac.1c01039

\section{Author Contributions}

A.D.-C. and L.R.-A. contributed equally. A.D.-C. and G.B. developed the concept and design of the project. Synthesis and physicochemical characterization, except for circular dichroism and all kinds of electron microscopy, were performed by A.D.C. Circular dichroism measurements were performed by C.N.J. Conventional TEM imaging was performed by L.R.-A. who also conducted the rheological measurements. Conventional STEM and LTEM imaging was performed by C.D.P. and L.R.P. Image denoising was performed by G.M. Analysis and interpretation of the data involved the contribution of all authors. Ultrastructure analysis was performed by G.B. The manuscript was written mainly by A.D.-C., L.R.-A., and G.B. with contributions from all other authors and revised by A.D.-
C., L.R.-A., and G.B. All authors have given approval to the final version of the manuscript.

\section{Notes}

The authors declare the following competing financial interest(s): G.B. and A.D-C. are inventors on a patent application to this work filed by UCL Business (no. WO2020225538 (A1) published 12 November 2020). The authors declare that they have no other competing interests.

\section{ACKNOWLEDGMENTS}

A.D.-C. and G.B. acknowledge the Royal Society (Newton International Fellowship scheme 2017-NF171487) and EU H2020 MSCA-IF-792957 SPeNTa-Brain. The authors thank the ERC for the consolidator award (CheSSTaG 769798), EPSRC Established Career Fellowship (EP/N026322/1) and Jeol and DENS Solutions for sponsoring LPR salary, and part of C.D.P. and G.M. studentships. L.R.-A. thanks the UK's ESPRC for funding her salary (Grant EP/N026322/1) and acknowledges support by the Spanish State Research Agency (Spanish Ministry of Science and Innovation) through Juan de la Cierva Incorporacion Fellowship (IJC2018-037951-I). The authors thank Prof. Modesto T. Lopez-Lopez (University of Granada, Spain) for helpful discussions about gel rheology, Dr. Inmaculada Conejos (CIPF, Valencia, Spain) for her help with the CD, and Gabriel Ing (University College London, UK) for his support with transmission electron microscopy.

\section{REFERENCES}

(1) Duro-Castano, A.; Conejos-Sánchez, I.; Vicent, M. J. PeptideBased Polymer Therapeutics. Polymers 2014, 6, 515-551.

(2) Salas-Ambrosio, P.; Tronnet, A.; Since, M.; Bourgeade-Delmas, S.; Stigliani, J.-L.; Vax, A.; Lecommandoux, S.; Dupuy, B.; Verhaeghe, P.; Bonduelle, C. Cyclic Poly( $\alpha$-peptoid)s by Lithium bis(trimethylsilyl)amide (LiHMDS)-Mediated Ring-Expansion Polymerization: Simple Access to Bioactive Backbones. J. Am. Chem. Soc. 2021, 143, 3697-3702.

(3) Lee, D. Y.; Rejinold, N. S.; Jeong, S. D.; Kim, Y. C. Stimuliresponsive polypeptides for biomedical applications. Polymers 2018, 10, 830-850.

(4) Carlsen, A.; Lecommandoux, S. Self-assembly of polypeptidebased block copolymer amphiphiles. Curr. Opin. Colloid Interface Sci. 2009, 14, 329-339.

(5) Cai, C.; Lin, J.; Lu, Y.; Zhang, Q.; Wang, L. Polypeptide selfassemblies: Nanostructures and bioapplications. Chem. Soc. Rev. 2016, $45,5985-6012$.

(6) Deming, T. J. Synthetic polypeptides for biomedical applications. Prog. Polym. Sci. 2007, 32, 858-875.

(7) Lu, H.; Wang, J.; Song, Z.; Yin, L.; Zhang, Y.; Tang, H.; Tu, C.; Lin, Y.; Cheng, J. Recent advances in amino acid N-carboxyanhydrides and synthetic polypeptides: Chemistry, self-assembly and biological applications. Chem. Commun. 2014, 50, 139-155.

(8) Deng, C.; Wu, J.; Cheng, R.; Meng, F.; Klok, H.-A.; Zhong, Z. Functional polypeptide and hybrid materials: Precision synthesis via $\alpha$-amino acid $\mathrm{N}$-carboxyanhydride polymerization and emerging biomedical applications. Prog. Polym. Sci. 2014, 39, 330-364.

(9) Sun, L.; Zheng, C.; Webster, T. J. Self-assembled peptide nanomaterials for biomedical applications: Promises and pitfalls. Int. J. Nanomed. 2017, 12, 73-86.

(10) Brendel, J. C.; Schacher, F. H. Block Copolymer Self-Assembly in Solution-Quo Vadis? Chem. - Asian J. 2018, 13, 230-239.

(11) Mai, Y.; Eisenberg, A. Self-assembly of block copolymers. Chem. Soc. Rev. 2012, 41, 5969-5985.

(12) Penfold, N. J. W.; Yeow, J.; Boyer, C.; Armes, S. P. Emerging Trends in Polymerization-Induced Self-Assembly. ACS Macro Lett. 2019, 8, 1029-1054. 
(13) Mane, S. R. Trending methods employed for polymerization induced self-assembly. New J. Chem. 2020, 44, 6690-6698.

(14) Canning, S. L.; Smith, G. N.; Armes, S. P. A Critical Appraisal of RAFT-Mediated Polymerization-Induced Self-Assembly. Macromolecules 2016, 49, 1985-2001.

(15) Blanazs, A.; Madsen, J.; Battaglia, G.; Ryan, A. J.; Armes, S. P. Mechanistic insights for block copolymer morphologies: How do worms form vesicles? J. Am. Chem. Soc. 2011, 133, 16581-16587.

(16) Lv, F.; An, Z.; Wu, P. Scalable preparation of alternating block copolymer particles with inverse bicontinuous mesophases. Nat. Commun. 2019, 10, No. 1397.

(17) Warren, N. J.; Armes, S. P. Polymerization-induced selfassembly of block copolymer nano-objects via RAFT aqueous dispersion polymerization. J. Am. Chem. Soc. 2014, 136, 1017410185.

(18) D’Agosto, F.; Rieger, J.; Lansalot, M. RAFT-Mediated Polymerization-Induced Self-Assembly. Angew. Chem., Int. Ed. 2020, 59, 8368-8392.

(19) Liu, C.; Hong, C.-Y.; Pan, C.-Y. Polymerization Techniques in Polymerization-Induced Self-Assembly (PISA). Polym. Chem. 2020, $11,3673-3689$.

(20) Foster, J. C.; Varlas, S.; Blackman, L. D.; Arkinstall, L. A.; O'Reilly, R. K. Ring-Opening Metathesis Polymerization in Aqueous Media Using a Macroinitiator Approach. Angew. Chem., Int. Ed. 2018, 57, 10672-10676.

(21) Varlas, S.; Foster, J. C.; O’Reilly, R. K. Ring-opening metathesis polymerization-induced self-assembly (ROMPISA). Chem. Commun. 2019, 55, 9066-9071.

(22) Hill, M. R.; Guégain, E.; Tran, J.; Figg, C. A.; Turner, A. C.; Nicolas, J.; Sumerlin, B. S. Radical Ring-Opening Copolymerization of Cyclic Ketene Acetals and Maleimides Affords Homogeneous Incorporation of Degradable Units. ACS Macro Lett. 2017, 6, 1071-1077.

(23) Wright, D. B.; Touve, M. A.; Adamiak, L.; Gianneschi, N. C. ROMPISA: Ring-Opening Metathesis Polymerization-Induced SelfAssembly. ACS Macro Lett. 2017, 6, 925-929.

(24) Cornel, E. J.; Jiang, J.; Chen, S.; Du, J. Principles and characteristics of polymerization-induced self-assembly with various polymerization techniques. CCS Chem. 2021, 3, 2104-2125.

(25) Jiang, J.; Zhang, X.; Fan, Z.; Du, J. Ring-Opening Polymerization of N-Carboxyanhydride-Induced Self-Assembly for Fabricating Biodegradable Polymer Vesicles. ACS Macro Lett. 2019, 8, 12161221.

(26) Grazon, C.; Salas-Ambrosio, P.; Ibarboure, E.; Buol, A.; Garanger, E.; Grinstaff, M. W.; Lecommandoux, S.; Bonduelle, C. Aqueous Ring-Opening Polymerization-Induced Self-Assembly (ROPISA) of N-Carboxyanhydrides. Angew. Chem., Int. Ed. 2020, 59, 622-626.

(27) Bartlett, P. D.; Jones, R. H. A Kinetic Study of the Leuchs Anhydrides in Aqueous Solution. I. J. Am. Chem. Soc. 1957, 79, 21532159.

(28) Bartlett, P. D.; Dittmer, D. C. A Kinetic Study of the Leuchs Anhydrides in Aqueous Solution. II. J. Am. Chem. Soc. 1957, 79, 2159-2160.

(29) Ježek, J.; Cooper, K.; Strich, R. Reactive Oxygen Species and Mitochondrial Dynamics: The Yin and Yang of Mitochondrial Dysfunction and Cancer Progression. Antioxidants 2018, 7, No. 13.

(30) Schumacker, P. T. Reactive Oxygen Species in Cancer: A Dance with the Devil. Cancer Cell 2015, 27, 156-157.

(31) Patten, D. A.; Germain, M.; Kelly, M. A.; Slack, R. S. Reactive oxygen species: Stuck in the middle of neurodegeneration. $J$. Alzheimer's Dis. 2010, 20, S357-S367.

(32) Mittal, M.; Siddiqui, M. R.; Tran, K.; Reddy, S. P.; Malik, A. B. Reactive oxygen species in inflammation and tissue injury. Antioxid. Redox Signaling 2014, 20, 1126-1167.

(33) Padgett, L. E.; Broniowska, K. A.; Hansen, P. A.; Corbett, J. A.; Tse, H. M. The role of reactive oxygen species and proinflammatory cytokines in type 1 diabetes pathogenesis. Ann. N. Y. Acad. Sci. 2013, $1281,16-35$.
(34) Kojda, G.; Harrison, D. Interactions between NO and reactive oxygen species: Pathophysiological importance in atherosclerosis, hypertension, diabetes and heart failure. Cardiovasc. Res. 1999, 43, $562-571$.

(35) Saravanakumar, G.; Kim, J.; Kim, W. J. Reactive-OxygenSpecies-Responsive Drug Delivery Systems: Promises and Challenges. Adv. Sci. 2017, 4, No. 1600124.

(36) Ballance, W. C.; Qin, E. C.; Chung, H. J.; Gillette, M. U.; Kong, $\mathrm{H}$. Reactive oxygen species-responsive drug delivery systems for the treatment of neurodegenerative diseases. Biomaterials 2019, 217, No. 119292.

(37) Ye, H.; Zhou, Y.; Liu, X.; Chen, Y.; Duan, S.; Zhu, R.; Liu, Y.; Yin, L. Recent Advances on Reactive Oxygen Species-Responsive Delivery and Diagnosis System. Biomacromolecules 2019, 20, 24412463.

(38) Rodriguez, A. R.; Kramer, J. R.; Deming, T. J. EnzymeTriggered Cargo Release from Methionine Sulfoxide Containing Copolypeptide Vesicles. Biomacromolecules 2013, 14, 3610-3614.

(39) Chang, H.; Li, C.; Huang, R.; Su, R.; Qi, W.; He, Z. Amphiphilic hydrogels for biomedical applications. J. Mater. Chem. B 2019, 7, 2899-2910.

(40) Yamada, S.; Ikkyu, K.; Iso, K.; Goto, M.; Endo, T. Facile synthesis of polymethionine oxides through polycondensation of activated urethane derivative of $\alpha$-amino acid and their application to antifouling polymer against proteins and cells. Polym. Chem. 2015, 6, $1838-1845$.

(41) Ruiz-Pérez, L.; Messager, L.; Gaitzsch, J.; Joseph, A.; Sutto, L.; Gervasio, F. L.; Battaglia, G. Molecular engineering of polymersome surface topology. Sci. Adv. 2016, 2, No. e1500948.

(42) Krull, A.; Buchholz, T. O.; Jug, F. In Noise2Void-Learning Denoising from Single Noisy Images, Proceedings of the IEEE Computer Society Conference on Computer Vision and Pattern Recognition 2019-June; IEEE Computer Society, 2019; pp 21242132.

(43) Endo, T.; Sudo, A. Well-defined, environment-friendly synthesis of polypeptides based on phosgene-free transformation of amino acids into urethane derivatives and their applications. Polym. Int. 2020, 69, 219-227.

(44) Yamada, S.; Koga, K.; Sudo, A.; Goto, M.; Endo, T. Phosgenefree synthesis of polypeptides: Useful synthesis for hydrophobic polypeptides through polycondensation of activated urethane derivatives of $\alpha$-amino acids. J. Polym. Sci., Part A: Polym. Chem. 2013, 51, 3726-3731.

(45) Kamei, Y.; Sudo, A.; Endo, T. Synthesis of polypeptide having defined terminal structures through polymerization of activated urethane-derivative of $\gamma$-benzyl-L-glutamate. Macromolecules 2008, 41, 7913-7919.

(46) Scionti, G.; Moral, M.; Toledano, M.; Osorio, R.; Duran, J. D. G.; Alaminos, M.; Campos, A.; Lopez-Lopez, M. T. Effect of hydration on the biomechanical properties in a fibrin-agarose tissuelike model. J. Biomed. Mater. Res., Part A 2014, 102, 2573.

(47) Suarez-Fernandez, W. R.; Scionti, G.; Duran, J. D. G.; Zubarev, A. Y.; Lopez-Lopez, M. T. Role of particle clusters on the rheology of magneto-polymer fluids and gels. Philos. Trans. R. Soc., A 2020, 378, No. 20190254.

(48) Shriky, B.; Kelly, A.; Isreb, M.; Babenko, M.; Mahmoudi, N.; Rogers, S.; Shebanova, O.; Snow, T.; Gough, T. Pluronic F127 thermosensitive injectable smart hydrogels for controlled drug delivery system development. J. Colloid Interface Sci. 2020, 565, 119.

(49) Pace, C. N.; Scholtz, J. M. A Helix Propensity Scale Based on Experimental Studies of Peptides and Proteins. Biophys. J. 1998, 75, $422-427$.

(50) Bromley, E. H.; Channon, K. J.; King, P. J.; Mahmoud, Z. N.; Banwell, E. F.; Butler, M. F.; Crump, M. P.; Dafforn, T. R.; Hicks, M. R.; Jonathan, D.; Hirst, J. D.; et al. Assembly Pathway of a Designed $\alpha$-Helical Protein Fiber. Biophys. J. 2010, 98, 1668-1676.

(51) Battaglia, G.; Ryan, A. J. Bilayers and Interdigitation in Block Copolymer Vesicles. J. Am. Chem. Soc. 2005, 127, 8757-8764. 
(52) Fernyhough, C.; Ryana, A. J.; Battaglia, G. pH controlled assembly of a polybutadiene-poly(methacrylic acid) copolymer in water: packing considerations and kinetic limitations. Soft Matter 2009, 5, 1674-1682.

(53) Battaglia, G.; Ryan, A. J. Effect of Amphiphile Size on the Transformation from a Lyotropic Gel to a Vesicular Dispersion. Macromolecules 2006, 39, 798-805.

(54) Battaglia, G.; Ryan, A. J. The evolution of vesicles from bulk lamellar gels. Nat. Mater. 2005, 4, 869-876.

(55) Forman, H. J.; Bernardo, A.; Davies, K. What is the concentration of hydrogen peroxide in blood and plasma? Arch. Biochem. Biophys. 2016, 603, 48-53.

(56) Panieri, E.; Santoro, M. M. ROS signaling and redox biology in endothelial cells. Cell. Mol. Life Sci. 2015, 72, 3281-3303.

(57) Drazic, A.; Winter, J. The physiological role of reversible methionine oxidation. Biochim. Biophys. Acta, Proteins Proteomics 2014, 1844, 1367-1382.

(58) Xu, Q.; He, C.; Ren, K.; Xiao, C.; Chen, X. Thermosensitive Polypeptide Hydrogels as a Platform for ROS-Triggered Cargo Release with Innate Cytoprotective Ability under Oxidative Stress. Adv. Healthcare Mater. 2016, 5, 1979-1990.

(59) Schneider, N. M.; Norton, M. M.; Mendel, B. J.; Grogan, J. M.; Ross, F. M.; Bau, H. H. Electron-Water interactions and implications for liquid cell electron microscopy. J. Phys. Chem. C 2014, 118, 22373-22382.

(60) Grogan, J. M.; Bau, H. H. The nanoaquarium: A platform for in situ transmission electron microscopy in liquid media. J. Microelectromech. Syst. 2010, 19, 885-894. 